\title{
Prevalence, Correlates and Outcomes of Gastric Antral Vascular Ectasia in Systemic Sclerosis: A EUSTAR Case-control Study
}

\author{
Etienne Ghrénassia, Jérome Avouac, Dinesh Khanna, Chris T. Derk, Oliver Distler, \\ Yossra Atef Suliman, Paolo Airo, Patricia E. Carreira, Rosario Foti, Brigitte Granel, \\ Alice Berezne, Jean Cabane, Francesca Ingegnoli, Edoardo Rosato, Paola Caramaschi, \\ Roger Hesselstrand, Ulrich A. Walker, Juan Jose Alegre-Sancho, Virginie Zarrouk, \\ Christian Agard, Valeria Riccieri, Elena Schiopu, Heather Gladue, Virginia D. Steen, \\ and Yannick Allanore
}

ABSTRACT. Objective. To estimate the prevalence, determine the subgroups at risk, and the outcomes of patients with systemic sclerosis (SSc) and gastric antral vascular ectasia (GAVE).

Methods. We queried the European League Against Rheumatism Scleroderma Trials and Research (EUSTAR) network for the recruitment of patients with SSc-GAVE. Each case was matched for cutaneous subset and disease duration with 2 controls with SSc recruited from the same center, evaluated at the time the index case made the diagnosis of GAVE. SSc characteristics were recorded at the time GAVE occurred and the last observation was collected to define the outcomes.

Results. Forty-nine patients with SSc and GAVE were included (24 with diffuse cutaneous SSc) and compared to 93 controls with SSc. The prevalence of GAVE was estimated at about $1 \%$ of patients with SSc. By multivariate analysis, patients with SSc-GAVE more frequently exhibited a diminished $(<75 \%)$ DLCO value (OR 12.8; 95\% CI 1.9-82.8) despite less frequent pulmonary fibrosis (OR 0.2; 95\% CI 0.1-0.6). GAVE was also associated with the presence of anti-RNA-polymerase III antibodies (OR 4.6; 95\% CI 1.2-21.1). SSc-GAVE was associated with anemia (82\%) requiring blood transfusion (45\%). Therapeutic endoscopic procedures were performed in $45 \%$ of patients with GAVE. After a median followup of 30 months (range 1-113 months), survival was similar in patients with SSc-GAVE compared to controls, but a higher number of scleroderma renal crisis cases occurred ( $12 \%$ vs $2 \%$; $\mathrm{p}=0.01)$.

Conclusion. GAVE is rare and associated with a vascular phenotype, including anti-RNA-polymerase III antibodies, and a high risk of renal crisis. Anemia, usually requiring blood transfusions, is a common complication. (First Release Dec 1 2013; J Rheumatol 2014;41:99-105; doi:10.3899/ jrheum.130386)

Key Indexing Terms:

SYSTEMIC SCLEROSIS RNA POLYMERASE III

WATERMELON STOMACH
SCLERODERMA RENAL CRISIS

From the Department of Rheumatology A, Paris Descartes University, Cochin Hospital, AP-HP, Paris, France; University of Michigan Scleroderma Program, Ann Arbor, Michigan, USA; Division of Rheumatology, University of Pennsylvania, Philadelphia, USA; Department of Rheumatology, University Hospital Zurich, Switzerland; Rheumatology and Clinical Immunology Unit, Spedali Civili and University of Brescia, Brescia, Italy; Rheumatology Department, Hospital Universitario 12 de Octubre, Madrid, Spain; Rheumatology Unit, Azienda Ospedaliera Universitaria V. Emanuele, Ferrarotto, S. Bambino, Catania, Italy; Service de Médecine Interne, Centre Hospitalier Nord, Marseille, France; Service de Médecine Interne, University Hospital Centre (CHU) Cochin; Service de Médecine Interne, CHU Saint-Antoine, Paris, France, Division of Rheumatology, Istituto Gaetano Pini, University of Milan, Italy; Department of Clinical Medicine, Clinical Immunology Unit, Sapienza University of Rome, Rome, Italy; Rheumatology Unit, Azienda Ospedaliera Universitaria Integrata, Verona, Italia; Department of Rheumatology, Lund University, Lund, Sweden; Department of Rheumatology, Basel University, Basel, Switzerland; Sección de Reumatología, Hospital Dr Peset, Valencia, Spain; Médecine interne,

\begin{abstract}
Hôpital Beaujon, Clichy, France; Internal Medicine, Hôtel-Dieu Hospital, CHU Nantes, Nantes, France; Rheumatologic Unit, Department of Internal Medicine and Clinical Specialties, Sapienza University of Rome, Rome, Italy; and Department of Medicine, Georgetown University Medical Center, Washington DC, USA.

Dr. Khanna was supported by US National Institutes of Health/US National Institute of Arthritis and Musculoskeletal and Skin Diseases K24 AR063120.

E. Ghrénassia, fellow; J. Avouac, MD, PhD, Department of Rheumatology A, Paris Descartes University, Cochin Hospital; D. Khanna, MD, University of Michigan Scleroderma Program, C.T. Derk, MD, Division of Rheumatology, University of Pennsylvania; O. Distler, MD, PhD; Y. Atef Suliman, MD, Department of Rheumatology, University Hospital Zurich; P. Airo, MD, Rheumatology and Clinical Immunology Unit, Spedali Civili and University of Brescia; P.E. Carreira, $M D$, Rheumatology Department, Hospital Universitario 12 de Octubre; R. Foti, MD, Rheumatology Unit, Azienda Ospedaliera Universitaria V. Emanuele; B. Granel, MD, PhD, Service de Médecine Interne, Centre Hospitalier Nord; A. Berezne, MD, Service de Médecine Interne, $C H U$
\end{abstract}


Cochin; J. Cabane, MD, Service de Médecine Interne, CHU SaintAntoine; F. Ingegnoli, MD, Division of Rheumatology, Istituto Gaetano Pini, University of Milan; E. Rosato, MD, Department Of Clinical Medicine, Clinical Immunology Unit, Sapienza University of Rome; P. Caramaschi, MD, Rheumatology Unit, Azienda Ospedaliera Universitaria Integrata; $R$. Hesselstrand, $M D$, Department of Rheumatology, Lund University; U.A. Walker, MD, PhD, Department of Rheumatology, Basel University; J.J. Alegre-Sancho, MD, Sección de Reumatología, Hospital Dr Peset; V. Zarrouk, MD, Médecine interne, Hôpital Beaujon; C. Agard, MD, Internal Medicine, Hôtel-Dieu Hospital; V. Riccieri, $M D$, PhD, Rheumatologic Unit, Department of Internal Medicine and Clinical Specialties, Sapienza University of Rome; E. Schiopu, MD; H. Gladue, MD, University of Michigan Scleroderma Program; V.D. Steen, MD, Department of Medicine, Georgetown University Medical Center; and Y. Allanore, MD, PhD, Department of Rheumatology A, Paris Descartes University, Cochin Hospital.

Address correspondence to Professor Y. Allanore, Service de Rhumatologie A, hôpital Cochin, 27 rue du faubourg St Jacques, 75014 Paris, France.E-mail: yannick.allanore@cch.aphp.fr

Accepted for publication September 12, 2013.

Watermelon stomach or gastric antral vascular ectasia (GAVE) is a rare event known to be associated with liver diseases and is thought to occur in about $2 \%$ of patients awaiting liver transplantation ${ }^{1}$. GAVE has a unique endoscopic appearance, characterized by multiple longitudinal stripes of red vessels, which radiate in a spoke-like fashion from the pylorus to the antrum. This characteristic appearance is reminiscent of the stripes of a watermelon, thus accounting for the term "watermelon stomach." The microscopic aspect is defined by dilatation of mucosal capillaries, focal fibrin thrombosis, fibromuscular hyperplasia, and fibrohyalinosis 2,3 .

GAVE can also be observed in patients in nonhepatic chronic diseases such as autoimmune connective tissue disorders ${ }^{4}$, bone marrow transplantation, chronic renal failure, and heart diseases. In systemic sclerosis (SSc), GAVE is a complication thought to belong to the spectrum of vascular alterations ${ }^{5}$. Despite the frequent use of gastroscopy, the prevalence of SSc-GAVE remains poorly known, with estimates reported to vary largely in the literature (from $1.8 \%$ to $22.3 \%)^{6,7,8,9}$. Because of the rarity of this disease manifestation, the clinical phenotype of patients with SSc-GAVE has not been well studied, although a variety of clinical associations have been reported ${ }^{5,6,10}$. In addition, the prognosis of GAVE still remains a matter of debate. The aim of our study was to clarify prevalence, SSc-associated features, and prognosis of GAVE, taking advantage of the large European League Against Rheumatism Scleroderma Trial and Research (EUSTAR) network.

\section{MATERIALS AND METHODS}

We queried the EUSTAR centers (182 centers worldwide) by e-mail for patients with SSc and a diagnosis of GAVE. We asked the centers that had at least 1 case identified to complete a dedicated data collection instrument and to provide disease characteristics according to the EUSTAR Minimal Essential Data Set (MEDS) online format ${ }^{11,12}$. This dedicated data collection instrument covered demographics, disease duration (first
non-Raynaud symptom), organ involvement, and laboratory data of SSc plus clinical presentation, treatment, and followup.

To estimate the point prevalence of symptomatic GAVE, we asked each participating center to provide the number of patients followed who had SSc. To further clarify the phenotype and outcomes, we asked each of the participating centers to match 2 patients with SSc seen at their center at the time of diagnosis of SSc-GAVE index case ( \pm 12 months) for cutaneous subset and disease duration, as we did in a previous EUSTAR study ${ }^{13}$. The same disease characteristics as for the GAVE cases were collected according to MEDS online.

Our study was approved by the clinical research committee of EUSTAR and approved by all local ethics committees.

Computer software (MedCalc, version 11.4.4; MedCalc Software bvba) was used for statistical analyses. Continuous values were reported as median (range). Continuous variables were compared with the Mann-Whitney U test. Categorical variables were reported as absolute number or percentage. Categorical variables were compared using the chi-square test, or Fisher's exact test, as appropriate. OR with their 95\% CI were calculated. Multivariate analysis was performed using stepwise logistic regression with backward elimination, which involves starting with all candidate variables, testing the deletion of each variable using a chosen model comparison criterion, deleting the variable (if any) that improves the model the most by being deleted, and repeating this process until no further improvement is possible. To be as inclusive as possible, and not to miss an important variable independently associated with GAVE, all variables with a univariate $\mathrm{p}$ value $\leq 0.1$ were entered as covariates in the stepwise regression analysis. For all study variables, 2 -tailed $\mathrm{p}$ values $<0.05$ were considered statistically significant.

\section{RESULTS}

Among 20 participating centers, we identified 49 patients with SSc-GAVE, with a diagnosis made by upper gastrointestinal (GI) endoscopy in all cases. The number of cases sent per center ranged from 1 to 7 . According to the estimated number of symptomatic patients followed by the participating centers, the estimated point prevalence was $1 \%$ (39/4090 patients). This prevalence did not take into account 6 asymptomatic patients with GAVE and 4 additional patients for whom the total number of patients with SSc followed in their center was missing. The prevalence ranged between $0.4 \%$ and $5 \%$ among the different centers.

Ninety percent of the patients with SSc-GAVE were females with a median age of 56 years (range 20-83 yrs). Twenty-four patients $(49 \%)$ had the diffuse cutaneous subset (dcSSc) and $25(51 \%)$ had the limited subset (lcSSc). The median delay between the first non-Raynaud symptom and the diagnosis of GAVE was 19 months (range 0-180 mos). It was 13 months (range $0-180$ mos) in patients with dcSSc and 24 months (range 0-114 mos) in patients with lcSSc $(p=0.63)$. The median delay between Raynaud and the diagnosis of GAVE was 38 months (range 0-396 mos). It was 16 months (range 0-192 mos) in patients with dcSSc and 60.5 months (range 0-396 mos), in patients with lcSSc $(\mathrm{p}=0.18)$.

Patients with SSc-GAVE were matched with 93 controls (5 controls were missing because 3 centers could not provide 2 expected matched controls per case). Thus, GAVE and control groups were similar for the median disease duration (19 mos vs 14 mos; $\mathrm{p}=0.2)$ and proportions of 
cutaneous subsets (49\% diffuse cutaneous in the GAVE patients vs $47 \%$ diffuse cutaneous in the matched controls; $\mathrm{p}=0.9)$.

We first determined factors associated with the presence of GAVE by univariate analyses. As illustrated in Table 1, patients with SSc-GAVE were significantly more likely to have systemic hypertension ( $45 \%$ vs $15 \%$; $=0.0002)$ and reduced $\mathrm{CO}$ diffusion $[\mathrm{DLCO} /$ alveolar volume $(\mathrm{AV})<75 \%$ predicted in $51 \%$ vs $27 \%$; $\mathrm{p}=0.008$ ], despite less lung fibrosis on thoracic computed tomography (CT) scan (22\% vs $44 \%$; $p=0.02$ ), defined by the presence of at least 1 usual sign of SSc-associated lung fibrosis, i.e., interstitial reticular pattern with or without traction bronchiectasis, and/or honeycomb cysts ${ }^{14,15}$. Of note, the capillaroscopic patterns were similar between the 2 groups $^{16}$. In addition, there was no association with other disorders suspected to be associated with GAVE, including cardiac, liver, renal insufficiency, pernicious anemia, primary biliary cirrhosis, and thyroiditis.

Because cases and controls were matched for the cutaneous subset, there was, as expected, no difference in anticentromere antibody status between the 2 groups. However, despite the fact that $51 \%$ of patients with SSc-GAVE had the diffuse cutaneous subset, it is striking that only 1 of them had antitopoisomerase I (anti-topo I) antibodies, while $35 \%$ of controls were anti-topo I positive $(p<0.0001)$. The other main finding was the higher prevalence of anti-RNA polymerase III in patients with SSc-GAVE (48\% vs 16\%; $\mathrm{p}=0.01$; Table 2).

We also compared the clinical characteristics of patients regarding the RNA polymerase III status. Patients with anti-RNA polymerase III antibodies were more likely to have significantly shorter disease duration $(\mathrm{p}=0.03)$ and significantly higher proportion of GAVE $(p=0.01)$ and scleroderma renal crisis $(\mathrm{p}=0.02$; Table 3$)$.

In multivariate analyses (Table 4), we identified the following factors that were independently associated with GAVE: a reduced frequency of lung fibrosis on chest CT scan (OR 0.2; 95\% CI 0.1-0.6), reduced DLCO/AV < 75\% predicted (OR 12.8; 95\% CI 1.9-82.8) and positive anti-RNA-polymerase III antibodies (OR 4.6; 95\% CI 1.2-21.1).

Among the 49 patients with SSc-GAVE, 6 (12\%) were asymptomatic and identified by systematic gastroscopy assessment. The main symptoms and clinical presentation of GAVE were anemia in 40 patients (82\%), upper GI bleeding in $5(10 \%)$, and lower GI bleeding in $2(4 \%)$. Twenty-two patients with SSc-GAVE (45\%) needed blood transfusions

Table 1. Comparison of clinical features between patients with systemic sclerosis (SSc) and gastric antral vascular ectasia (GAVE) and control patients with SSc in univariate analysis.

\begin{tabular}{|c|c|c|c|}
\hline Vascular Features & $\begin{array}{c}\text { Patients with SSc- } \\
\text { GAVE, n }(\%)\end{array}$ & $\begin{array}{c}\text { Patients with SSc } \\
\text { without GAVE, n (\%) }\end{array}$ & $\mathrm{p}$ \\
\hline \multicolumn{4}{|l|}{ Vasular features } \\
\hline \multicolumn{4}{|l|}{ Capillaroscopy features } \\
\hline Giant capillaries & $34(69)$ & $74(80)$ & 0.2 \\
\hline Avascular areas & $28(58)$ & $44(47)$ & 0.3 \\
\hline Active digital ulcers & $8(16)$ & $17(18)$ & 0.9 \\
\hline History of digital ulcers & $21(42)$ & $30(32)$ & 0.4 \\
\hline History + active digital ulcers & $23(47)$ & $31(33)$ & 0.1 \\
\hline PAH confirmed by RHC & $4(9)$ & $5(5)$ & 0.6 \\
\hline Systemic hypertension & $22(45)$ & $14(15)$ & 0.0002 \\
\hline \multicolumn{4}{|l|}{ Lung involvement } \\
\hline Pulmonary fibrosis on CT scan & $11(22)$ & $41(44)$ & 0.02 \\
\hline FVC $<75 \%$ predicted & $7(14)$ & $22(24)$ & 0.2 \\
\hline $\mathrm{DLCO} / \mathrm{AV}<75 \%$ predicted & $25(51)$ & $25(27)$ & 0.008 \\
\hline \multicolumn{4}{|l|}{ Digestive involvement } \\
\hline Gastroesophageal reflux & $37(76)$ & $68(73)$ & 0.9 \\
\hline Digestive malabsorption & $4(8)$ & $11(12)$ & 0.7 \\
\hline Treated microbial overgrowth & $2(4)$ & $8(9)$ & 0.5 \\
\hline Anorectal incontinence & $3(6)$ & $1(1)$ & 0.2 \\
\hline \multicolumn{4}{|l|}{ Treatment } \\
\hline Immunosuppressors & $23(47)$ & $56(60)$ & 0.2 \\
\hline Cyclophosphamide & $7(14)$ & $17(18)$ & 0.7 \\
\hline Azathioprine & $0(0)$ & $7(8)$ & 0.1 \\
\hline Methotrexate & $8(16)$ & $20(22)$ & 0.6 \\
\hline Mycophenolate mofetil & $8(16)$ & $12(13)$ & 0.8 \\
\hline Other & $7(14)$ & $7(8)$ & 0.4 \\
\hline Corticosteroids $>10 \mathrm{mg} /$ day & $4(8)$ & $9(10)$ & 0.9 \\
\hline
\end{tabular}

PAH: pulmonary arterial hypertension; RHC: right heart catheterization; CT: computed tomography; FVC: forced vital capacity; AV: alveolar volume. 
Table 2. Comparison of antibody status between patients with systemic sclerosis (SSc) and gastric antral vascular ectasia (GAVE) and control patients with SSc in univariate analysis.

\begin{tabular}{lccc}
\hline Antibody Status & $\begin{array}{c}\text { Patients with } \\
\text { SSc-GAVE, n (\%) }\end{array}$ & $\begin{array}{c}\text { Patients with SSc } \\
\text { without GAVE, n (\%) }\end{array}$ & $\mathrm{p}$ \\
\hline Positive antinuclear antibodies & $44(90)$ & $89(96)$ & 0.3 \\
Positive antitopoisomerase I antibodies & $1(2)$ & $32(34)$ & $<0.0001$ \\
Positive anticentromere antibodies & $17(34)$ & $25(27)$ & 0.5 \\
Positive RNA polymerase III antibodies & $12(48)^{*}$ & $7(16)^{*}$ & 0.01 \\
\hline
\end{tabular}

*RNA polymerase III antibodies were only available for 25 patients with GAVE and 43 patients without GAVE.

Table 3. Patient characteristics according to anti-RNA polymerase III antibody status.

\begin{tabular}{lccc}
\hline & $\begin{array}{c}\text { Positive Anti-RNA } \\
\text { Polymerase III } \\
\text { Antibodies, } \\
\mathrm{n}=19\end{array}$ & $\begin{array}{c}\text { Negative Anti-RNA } \\
\text { Polymerase III } \\
\text { Antibodies, } \\
\mathrm{n}=49\end{array}$ & $\mathrm{p}$ \\
\hline Diffuse cutaneous subset, $\mathrm{n}(\%)$ & $13(68)$ & $24(49)$ & 0.2 \\
Disease duration, yrs, median (range) & $8(0-114)$ & $22(0-180)$ & 0.03 \\
GAVE, n (\%) & $12(63)$ & $13(27)$ & 0.01 \\
Scleroderma renal crisis, $\mathrm{n}(\%)$ & $4(21)$ & $1(2)$ & 0.02 \\
\hline
\end{tabular}

GAVE: gastric antral vascular ectasia.

Table 4. Comparison of clinical and immunological items between patients with systemic sclerosis (SSc) and gastric antral vascular ectasia (GAVE) and control patients with SSc using multivariate analysis (stepwise logistic regression including disease characteristics with a univariate $\mathrm{p} \leq 0.1$ ).

\begin{tabular}{lccc}
\hline Clinical and Immunologic Items & Univariate $\mathrm{p}$ & Multivariate $\mathrm{p}$ & $\begin{array}{c}\text { OR } \\
(95 \% \mathrm{CI})\end{array}$ \\
\hline History and active digital ulcers & & & $\mathrm{NA}$ \\
Systemic hypertension & 0.1 & Not significant & $\mathrm{NA}$ \\
Pulmonary fibrosis on CT scan & 0.0002 & Not significant & $0.2(0.1-0.6)$ \\
DLCO/AV < 75\% & 0.02 & 0.01 & $12.8(1.98-82.76)$ \\
Antitopoisomerase I antibodies & 0.008 & 0.007 & $\mathrm{NA}$ \\
Anti-RNA polymerase III antibodies & 0.0001 & Not significant & \multirow{2}{*}{$0.62(1.2-21.12)$} \\
\hline
\end{tabular}

NA: not applicable; CT: computed tomography; AV: alveolar volume.

of a mean of 2 units of packed red cells. Among them, 4 were transfused with more than 10 units of packed red cells. Twenty-eight patients $(57 \%)$ required a therapeutic endoscopic procedure, with argon plasma coagulation $(63 \%)$ or laser yttrium aluminum garnet (YAG; 30\%) in most cases. Two patients (4\%) requested gastric hemostatic surgery. After a median followup of 30 months (range 1-113 mos), 17 patients (35\%) had a relapse of bleeding requiring new local endoscopic treatment. Eight needed new red blood cell transfusions. There were 6 deaths from any cause $(12 \%)$ in the SSc-GAVE group and 6 deaths $(6.2 \%)$ in the SSc-control group $(\mathrm{p}=0.45)$. Of the most interest, 6 cases $(12 \%)$ of scleroderma renal crisis in the SSc-GAVE group occurred during the followup period, compared to 2 cases $(2 \%)$ in the control group $(\mathrm{p}=0.01)$. Of note, 3 of the 6 patients with GAVE and 1 of the 2 controls who developed scleroderma renal crisis had positive anti-RNA polymerase III antibodies.

\section{DISCUSSION}

This study is the largest series of GAVE in patients with SSc. It reflects the experience of a large number of participating centers and was not influenced by only a few of them (the number of cases sent per center ranged from 1 to 7). Nevertheless, despite querying a large network we were only able to assemble a series of 49 cases, which provides an estimated prevalence of symptomatic GAVE at about $1 \%$. This frequency is consistent with the identification of $2 / 110$ patients $(2 \%)$ with GAVE in a previous study that performed a systematic endoscopic assessment for the risk of Barret esophagus ${ }^{17}$. However, this prevalence is much lower than in some previous series. Indeed, a recent study showed a 
prevalence of $22.3 \%$ of GAVE in patients with severe early diffuse SSc recruited in a trial of autologous stem cell transplantation (Scleroderma Cyclophosphamide Or Transplant, SCOT, trial), where all patients regardless of clinical symptoms were assessed by using systematic upper GI endoscopy ${ }^{9}$. The inclusion of unselected patients with SSc, reflecting routine care and current practice, the retrospective collection of data, and the absence of systematic endoscopic assessment for all EUSTAR patients, leading to missed GAVE cases, may also account for the lower prevalence of GAVE in our sample.

Only a systematic endoscopic evaluation, which was beyond the scope of our study, could give the real prevalence of GAVE in patients with SSc. In addition, GAVE is sometimes difficult to recognize endoscopically, and thus may be missed or misdiagnosed by routine endoscopy. This was highlighted by a study that identified with the use of videocapsule 6/128 patients (4.7\%) with GAVE missed by routine endoscopy ${ }^{18}$. However, the use of videocapsule remains a matter of debate in SSc because of potential safety issues.

The diagnosis of GAVE was made 19 months after the diagnosis of SSc, in line with 2 previous studies that reported a median duration of 10 (range 6-168) and 36 (range 2-124) months ${ }^{6,10}$. Therefore, it seems to be an early complication, which might even occur earlier in patients with diffuse $\mathrm{SSc}^{10}$. It is noteworthy that GAVE was the first non-Raynaud event in 15 of our cases (30.6\%), consistently with data previously reported ${ }^{6}$. Several conditions, related or not to SSc, have also been suggested to be associated with GAVE, including cardiac, liver, renal insufficiency, pernicious anemia, primary biliary cirrhosis, and thyroiditis ${ }^{4,19}$, but we could not confirm these findings.

In the general population, vasodilating and angiogenic mediators; other humoral factors, such as vasoactive intestinal peptide; mechanical stress; and abnormal antral motility are associated with the presence of $\mathrm{GAVE}^{20}$. In SSc, GAVE is thought to be part of generalized microangiopathy that includes major disturbances of vascular mediators ${ }^{21}$. Indeed, similar histopathologic lesions can be found both in dermis and GAVE in SSc, such as capillary dilatation, small vessel fibrin deposition, and microthrombosis $^{5}$. Further, some authors reported lesions in other locations in SSc, such as rectal vascular ectasia ${ }^{22}$. Herein, we provide some evidence of the link between GAVE and vasculopathy, by identifying several factors related to SSc-associated vasculopathy associated with GAVE, including renal crisis, systemic hypertension, and reduced DLCO/AV.

We did not observe associated digestive comorbidities and, although previous reports suggested that cutaneous vascular lesions might be associated with GAVE, we could not evaluate cutaneous telangiectasia, because we were limited by missing data to perform a quantitative or semiquantitative approach to assess this vascular feature ${ }^{6,10}$.

Antinuclear antibodies (ANA) are found in over $90 \%$ of patients with SSc. Further, the presence of autoantibodies and their specificity is associated with disease progression and outcomes ${ }^{23}$. Positivity for 1 type of ANA usually remains throughout the course of the disease, and each subtype is usually exclusive of the others ${ }^{24}$. Our results highlight the scarcity of anti-topo I in patients with GAVE, which confirms previously reported data that showed a very low prevalence of anti-topo I antibodies among patients with GAVE, despite the high prevalence of the diffuse cutaneous form ${ }^{5,6,10}$. Indeed, Watson, et al $^{5}$ and Marie, et $a l^{6}$ reported no patient with anti-topo I antibody out of 5 and 15 patients, respectively, and Ingraham, et al reported only 1 positive patient out of $28^{10}$. In the SCOT trial, a trend was also observed for decreased frequency of topoisomerase antibodies in patients with GAVE (18.8\% vs $44.7 \%$; $\mathrm{p}=$ $0.071)$.

Of the most interest, positive anti-RNA polymerase III antibodies were independently associated with GAVE in our study sample, consistent with previous reports ${ }^{25}$. Moreover, the systematic followup of patients with GAVE allowed the identification of a higher risk of renal crisis in this patient subset, which is the master established risk of anti-RNA polymerase III antibodies. These data are in accordance with those of Ingraham, et $a l^{10}$ and Marie, et $a l^{6}$, who reported an unexpected high risk of renal crisis $(14 \%$ and $20 \%$, respectively) in their series. This further underlines the relationships between GAVE and anti-RNA polymerase III. Then, the presence of anti-RNA polymerase III seems to be a risk factor for several vascular events, such as GAVE, renal involvement, and also vascular lung or heart involvement, as suggested by the low DLCO/VA despite the absence of infiltrative lung disease. However, no association was observed between anti-RNA polymerase III and GAVE in the SCOT trial ${ }^{9}$; this might be explained by a selection bias, because our study included only patients with severe diffuse SSc. It is noteworthy that RNA polymerase III antibodies were not available from all patients in our cohort, because the detection of these antibodies is not yet performed in routine care in all the EUSTAR centers. The distribution of missing data regarding RNA polymerase III antibodies was similar between cases and controls, supporting that these antibodies were not obtained to specifically confirm their association with GAVE. Moreover, disease duration and cutaneous subset were not statistically different between patients with RNA polymerase III antibody information and patients without, supporting the absence of major ascertainment bias for obtaining RNA polymerase III information.

Very few series have looked at the clinical presentation of SSc-GAVE. In our series, a large proportion of patients (88\%) had clinically significant bleeding with anemia, often

Personal non-commercial use only. The Journal of Rheumatology Copyright @ 2014 . All rights reserved. 
requesting red blood cell transfusions. Accordingly, intervention therapies were used in half the patients. Argon plasma and Nd-YAG laser were the most frequently used, with good efficiency, although there was 35\% relapse and request for new endoscopic intervention and sometimes new transfusions. Indeed, treatment of GAVE is known to be led by hemostatic endoscopic procedures. Argon plasma seems to be the first choice because it has been the most reported in GAVE, has shown a high efficacy $(80 \%$ to $100 \%)$ with 2-4 sessions ${ }^{26,27}$, and has the lowest morbidity ${ }^{18}$. Nd-YAG laser can also be a useful treatment with $50 \%$ to $80 \%$ success rates reported ${ }^{17}$. However, relapses can occur and then treatment needs to be repeated. Cryotherapy and use of banding in the stomach antrum have also been tested in GAVE. Hemostatic surgery such as antrectomy is effective with no recurrence of bleeding, but a postoperative mortality rate of $6.6 \%$ was reported ${ }^{28}$. Various other therapies were used, including repeated blood transfusion, estrogen-progesterone treatment, octreotide, and steroids, but there is today no confirmation study in large populations ${ }^{17}$. In SSc-GAVE refractory disease, some case reports suggested that cyclophosphamide could be helpful ${ }^{29,30}$, but this remains to be evaluated.

The followup of patients with SSc-GAVE did not reach difference with controls in terms of mortality within the limited followup. However, we observed recurrences of bleeding and the development of renal crisis in patients with GAVE, demonstrating its severity in terms of morbidity. A longer followup of a larger number of patients is required to better evaluate the potential effect of GAVE on mortality.

GAVE is an infrequent complication of SSc commonly revealed by GI bleeding. Patients with SSc and iron deficiency, anemia, or GI bleeding should be strongly considered for GAVE. Thereafter, endoscopy should be accurately performed to make the right diagnosis and decide whether local therapy is needed. Patients with SSc and positive anti-RNA polymerase III are at high risk of GAVE, such as scleroderma renal crisis, which seems to belong to the same SSc vascular spectrum.

\section{REFERENCES}

1. Ward EM, Raimondo M, Rosser BG, Wallace MB, Dickson RD. Prevalence and natural history of gastric antral vascular ectasia in patients undergoing orthotopic liver transplantation. J Clin Gastroenterol 2004;38:898-900.

2. Suit PF, Petras RE, Bauer TW, Petrini JL Jr. Gastric antral vascular ectasia. A histologic and morphometric study of "the watermelon stomach". Am J Surg Pathol 1987;11:750-7.

3. Jabbari M, Cherry R, Lough JO, Daly DS, Kinnear DG, Goresky CA. Gastric antral vascular ectasia: the watermelon stomach. Gastroenterology 1984;87:1165-70.

4. Gostout CJ, Viggiano TR, Ahlquist DA, Wang KK, Larson MV, Balm R. The clinical and endoscopic spectrum of the watermelon stomach. J Clin Gastroenterol 1992;15:256-63.

5. Watson M, Hally RJ, McCue PA, Varga J, Jimenez SA. Gastric antral vascular ectasia (watermelon stomach) in patients with systemic sclerosis. Arthritis Rheum 1996;39:341-6.
6. Marie I, Ducrotte P, Antonietti M, Herve S, Levesque H. Watermelon stomach in systemic sclerosis: its incidence and management. Aliment Pharmacol Ther 2008;28:412-21.

7. Marie I, Levesque H, Ducrotte P, Denis P, Hellot MF, Benichou J, et al. Gastric involvement in systemic sclerosis: a prospective study. Am J Gastroenterol 2001;96:77-83.

8. Laoubi K, Allanore Y, Chaussade S, Kahan A, Wipff J. Estomac pastèque dans la sclérodermie systémique (in French). Watermelon stomach in systemic sclerosis. J Mal Vasc 2010;35:250-3.

9. Hung EW, Mayes MD, Sharif R, Assassi S, Machicao VI, Hosing $\mathrm{C}$, et al. Gastric antral vascular ectasia and its clinical correlates in patients with early diffuse systemic sclerosis in the SCOT trial. J Rheumatol 2013;40:455-60.

10. Ingraham KM, O’Brien MS, Shenin M, Derk CT, Steen VD. Gastric antral vascular ectasia in systemic sclerosis: demographics and disease predictors. J Rheumatol 2010;37:603-7.

11. Walker UA, Tyndall A, Czirjak L, Denton C, Farge-Bancel D, Kowal-Bielecka O, et al. Clinical risk assessment of organ manifestations in systemic sclerosis: a report from the EULAR Scleroderma Trials And Research group database. Ann Rheum Dis 2007;66:754-63.

12. Meier FM, Frommer KW, Dinser R, Walker UA, Czirjak L, Denton $\mathrm{CP}$, et al. Update on the profile of the EUSTAR cohort: an analysis of the EULAR Scleroderma Trials and Research group database. Ann Rheum Dis 2012;71:1355-60.

13. Allanore Y, Meune C, Vonk MC, Airo P, Hachulla E, Caramaschi P, et al. Prevalence and factors associated with left ventricular dysfunction in the EULAR Scleroderma Trial and Research group (EUSTAR) database of patients with systemic sclerosis. Ann Rheum Dis 2010;69:218-21.

14. Desai SR, Veeraraghavan S, Hansell DM, Nikolakopolou A, Goh NS, Nicholson AG, et al. CT features of lung disease in patients with systemic sclerosis: comparison with idiopathic pulmonary fibrosis and nonspecific interstitial pneumonia. Radiology 2004;232:560-7.

15. Goh NS, Desai SR, Veeraraghavan S, Hansell DM, Copley SJ, Maher TM, et al. Interstitial lung disease in systemic sclerosis: a simple staging system. Am J Respir Crit Care Med 2008; 177:1248-54.

16. Cutolo M, Sulli A, Pizzorni C, Accardo S. Nailfold videocapillaroscopy assessment of microvascular damage in systemic sclerosis. J Rheumatol 2000;27:155-60.

17. Wipff J, Allanore Y, Soussi F, Terris B, Abitbol V, Raymond J, et al Prevalence of Barrett's esophagus in systemic sclerosis. Arthritis Rheum 2005;52:2882-8.

18. Sidhu R, Sanders DS, McAlindon ME. Does capsule endoscopy recognise gastric antral vascular ectasia more frequently than conventional endoscopy? J Gastrointestin Liver Dis 2006;15:375-7.

19. Fuccio L, Mussetto A, Laterza L, Eusebi LH, Bazzoli F. Diagnosis and management of gastric antral vascular ectasia. World $\mathrm{J}$ Gastrointest Endosc 2013;5:6-13

20. Ripoll C, Garcia-Tsao G. The management of portal hypertensive gastropathy and gastric antral vascular ectasia. Dig Liver Dis 2011;43:345-51.

21. Avouac J, Meune C, Ruiz B, Couraud PO, Uzan G, Boileau C, et al. Angiogenic biomarkers predict the occurrence of digital ulcers in systemic sclerosis. Ann Rheum Dis 2012;71:394-9.

22. Singh D, Shill M, Kaur H. The watermelon rectum. J Clin Gastroenterol 2001;33:164-6.

23. Steen VD. Autoantibodies in systemic sclerosis. Semin Arthritis Rheum 2005;35:35-42.

24. Meyer O, De Chaisemartin L, Nicaise-Roland P, Cabane J, Tubach F, Dieude P, et al. Anti-RNA polymerase III antibody prevalence and associated clinical manifestations in a large series of French patients with systemic sclerosis: a cross-sectional study. 
J Rheumatol 2010;37:125-30.

25. Ceribelli A, Cavazzana I, Airo P, Franceschini F. Anti-RNA polymerase III antibodies as a risk marker for early gastric antral vascular ectasia (GAVE) in systemic sclerosis. J Rheumatol 2010;37:1544.

26. Lecleire S, Ben-Soussan E, Antonietti M, Goria O, Riachi G, Lerebours E, et al. Bleeding gastric vascular ectasia treated by argon plasma coagulation: a comparison between patients with and without cirrhosis. Gastrointest Endosc 2008;67:219-25.

27. Yusoff I, Brennan F, Ormonde D, Laurence B. Argon plasma coagulation for treatment of watermelon stomach. Endoscopy 2002;34:407-10.
28. Novitsky YW, Kercher KW, Czerniach DR, Litwin DE. Watermelon stomach: pathophysiology, diagnosis, and management. J Gastrointest Surg 2003;7:652-61.

29. Lorenzi AR, Johnson AH, Davies G, Gough A. Gastric antral vascular ectasia in systemic sclerosis: complete resolution with methylprednisolone and cyclophosphamide. Ann Rheum Dis 2001;60:796-8.

30. Schulz SW, O’Brien M, Maqsood M, Sandorfi N, Del Galdo F, Jimenez SA. Improvement of severe systemic sclerosis-associated gastric antral vascular ectasia following immunosuppressive treatment with intravenous cyclophosphamide. J Rheumatol 2009;36:1653-6. 